\title{
Crosscheck Principle in Pediatric Audiology Today: A 40-Year Perspective
}

\author{
James W. Hall III \\ Osborne College of Audiology, Salus University, Elkins Park, PA, \\ Department of Communication Sciences and Disorders, University of Hawaii, Honolulu, HI, USA, \\ Department of Audiology \& Speech Pathology, University of Pretoria, South Africa
}

Received July 1, 2016

Revised July 13, 2016

Accepted July 14, 2016

\section{Address for correspondence James W. Hall III, PhD \\ 371 Wooded Crossing Circle, St. Augustine, FL, USA \\ Tel +1-352-275-6335 \\ E-mail jwhall3phd@gmail.com}

The crosscheck principle is just as important in pediatric audiology as it was when first described 40 years ago. That is, no auditory test result should be accepted and used in the diagnosis of hearing loss until it is confirmed or crosschecked by one or more independent measures. Exclusive reliance on only one or two tests, even objective auditory measures, may result in a auditory diagnosis that is not clear or perhaps incorrect. On the other hand, close and careful analysis of findings for a test battery consisting of objective procedures and behavioral tests whenever feasible usually leads to prompt and accurate diagnosis of auditory dysfunction. This paper provides a concise review of the crosscheck principle from its introduction to its clinical application today. The review concludes with a description of a modern test battery for pediatric hearing assessment that supplements traditional behavioral tests with a variety of independent objective procedures including aural immittance measures, otoacoustic emissions, and auditory evoked responses.

J Audiol Otol 2016;20(2):59-67

KEY WORDS: Auditory brainstem response $\cdot$ Auditory steady state response Aural immittance measures $\cdot$ Crosscheck principle $\cdot$ Otoacoustic emissions.

\section{The Crosscheck Principle Revisited}

\section{Origin of the cross check principle}

Forty years ago, in 1976, James Jerger and Deborah Hayes first articulated the enduring "cross-check principle." At the time, Dr. Jerger was Director of Audiology at Methodist Hospital and Baylor College of Medicine in Houston Texas USA and Deborah Hayes was clinic supervisor and a $\mathrm{PhD}$ student. During this era, having just made the transition from speech pathology to audiology, I was working in the same clinic under the direction of Dr. Jerger and the supervision of Deborah Hayes.

In their classic paper entitled "The Cross-Check Principle in Pediatric Audiometry," Jerger and Hayes [1] illustrate viv-

This is an Open Access article distributed under the terms of the Creative Commons Attribution Non-Commercial License (http://creativecommons.org/licenses/by-nc/3.0/) which permits unrestricted non-commercial use, distribution, and reproduction in any medium, provided the original work is properly cited. idly with presentation of 5 case studies the limitations and pitfalls associated with exclusive reliance on behavioral test results. The first case was a 15 -year-old male with the diagnosis of severe-to-profound hearing loss when evaluated with an appropriate test battery. The hearing loss was identified at age 4-years, but then a subsequent hearing assessment at a university clinic appeared to show normal hearing and, sadly, the child was placed in a community home with the diagnosis of autism where he remained until for 7 years until his severe hearing loss was finally defined at age 15 years with test battery that included immittance measures with acoustic reflexes. The next case represents a scenario encountered all to often in clinical audiology. Over the course of 1.5 years, an otologist treated a young boy ( 2 years 9 months) for recurrent ear infections as mother became increasingly concerned about the delay in speech and language development. Three hearing assessments appeared to show a moderate conductive hearing loss. Apparently there was an assumption that hearing sensitivity would be normal with effective man- 
agement of the middle ear disease. Unfortunately, test results reported in the article for an evaluation with a battery of behavioral and independent objective tests, including auditory brainstem response (ABR), confirmed a severe mixed hearing loss with a substantial permanent sensory component.

The third child reported in the paper had undergone four hearing assessments at other facilities beginning at age 3.5 years, due mostly to the parents concerns about hearing status. Results were always different, ranging from an apparent severe hearing loss, normal hearing sensitivity, untestable, and a severe hearing loss bilaterally, greater for the left ear. Hearing aids and a preschool program for children with hearing impairment were recommended based on the last of these assessments. Results of the final assessment were described in detail in the article. Behavioral test findings suggesting normal hearing sensitivity were supplemented with a prediction of hearing sensitivity with the acoustic reflex and ABR thresholds within normal limits.

Case 4 was a child who had a severe seizure at 2.5 years with resulting neurological dysfunction that persisted for a year. Three hearing assessments thereafter appeared to show a rapid decrease in hearing thresholds from normal hearing to a profound hearing loss. Behavioral audiometry in the final assessment did suggest a profound hearing loss but acoustic reflex and ABR findings were consistent with normal hearing sensitivity. The final diagnosis was reportedly "auditory agnosia." The last case presented in the article was a 6-month old girl with aural atresia who yielded no behavioral response to sound a maximum intensity levels. ABR, however, confirmed a severe mostly conductive hearing loss bilaterally subsequently confirmed at age two years with pure tone audiometry.

Based on these clinical experiences, Jerger and Hayes [1] conclude their article with a strong argument for the use of a test battery consisting of independent test procedures, summarized in Table 1. The authors principally emphasized the value of applying aural immittance, then referred to as impedance audiometry, measures, and ABR (then called brainstem evoked response or BSER) to verify or "cross-check" the behavioral test results. Close inspection of the procedures in Table 1 reveals some limitations of technology available back in the 1970s. Tympanometry was performed only with a low frequency (220 or $226 \mathrm{~Hz}$ ) probe tone. Most clinical instrumentation then did not permit middle ear measurements with a high frequency probe tone. Also, acoustic reflexes could only be recorded with contralateral stimulation. A technological solution had not yet been found for simultaneously presenting a stimulus while also measuring in the same ear the acoustic reflex.
Table 1. Original battery of independent auditory tests described in the crosscheck article [1]

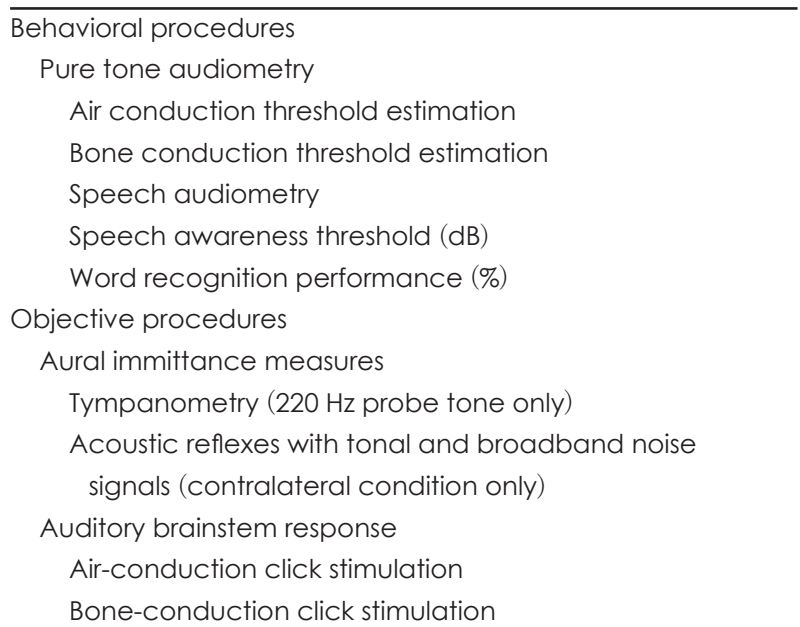

For several decades following its discovery, clinical ABR was elicited only with click stimulation. Clinical instrumentation did not include tone burst stimuli with appropriate windowing (ramping). This limitation is rather surprising since Jewett and Williston [2] in the original paper on ABR clearly demonstrated the feasibility of evoking the response with tone burst stimuli. Of course, a variety of equipment advances and features, like insert earphones and chirp stimuli, were not even envisioned when the crosscheck article was published. Likewise, the auditory steady state response (ASSR) technique had not been discovered. These technological advances in auditory assessment are reviewed later in this paper.

\section{What about electrocochleography (ECochG) and cortical auditory evoked responses?}

ECochG and cortical auditory evoked responses were well known in the 1970 s as these electrophysiological measures were first reported many years earlier in the 1930s. It is reasonable to question why the original test battery described in the crosscheck principle paper didn't include these electrophysiological auditory procedures. The simple answer is that very few clinics applied ECochG or cortical auditory evoked responses in pediatric auditory assessment. However, both types of electrophysiological procedures were available in the Methodist Hospital and Baylor College of Medicine where the patients in the crosscheck article underwent evaluation.

We'll consider cortical auditory evoked responses first. Back in the 1960s and early 1970s, before the first publications on ABR, Jerger, et al. [3,4]. Jerger and Jerger [5] published several articles describing the clinical value of the auditory late response in diagnosis of central auditory nervous system dysfunction. Instrumentation for measurement of cortical evoked responses was on hand in the at the time when 
the children highlighted in the cross-check were evaluated. Why weren't cortical responses included in the pediatric test battery? The disadvantages of the late auditory evoked response as a tool for auditory threshold estimation in young or difficult-to-test children are well appreciated [6]. A quiet patient state with minimal movement interference is required for confident detection of late responses. Unfortunately, cortical evoked responses are suppressed or eliminated with sedation or anesthesia, and even natural sleep markedly affects reliable measurement of cortical responses. Nonetheless, recent research suggests that cortical auditory evoked responses can play a role in the assessment and management of infant hearing loss, as noted in a later section of this article.

There are two related explanations for why ECochG was not included in the pediatric test battery described in the original crosscheck paper. One explanation had to do with electrode options. In the early 1970s, ECochG recording was performed with invasive surgical techniques involving transtympanic insertion of a needle onto the promontory or placement of a small silver ball in the round window niche following a myringotomy. Non-invasive electrode options, such as tympanic membrane or external ear canal electrodes (e.g., TIPtrodes) were not yet available for clinical use. Therefore, routine measurement of ECochG in an audiology clinic without anesthesia and otolaryngology support was clearly not feasible.

Interestingly, another explanation for the conspicuous absence of ECochG from the pediatric auditory test battery was more specific to the organization of the clinics in the Department of Otolaryngology at Baylor College of Medicine and Methodist Hospital. The audiology clinics under the direction of Dr. Jerger focused on hearing assessment of children and adults. The focus was diagnosis of auditory dysfunction and management of patients with communication disorders. Patients with vestibular and balance disorders were evaluated in another separate clinic under the direction of an otolaryngologist who enjoyed a very good reputation for his innovative neurophysiological research, Dr. Alfred Coats.

Some readers might not appreciate the important role that Dr. Coats played in documenting the clinical value of a variety of specific vestibular test procedures and contributing to the development the electronystagmography battery as evidenced by a series of publications from the 1960s into the 1970s [7-10]. Toward the end of this era Dr. Coats also published original research on ECochG [11,12] including some of the first papers describing non-surgical ECochG measurement with a special butterfly-design external ear canal electrode that was later commercially available and known as the "Coats electrode" [13]. Not unexpectedly, his research inter- ests led to some of the first studies demonstrating abnormally enhanced summating potential as an ECochG finding in patients with diagnosed Meniere's disease [14].

Thus, during this time frame, children underwent electrophysiological assessment of hearing in the audiology clinic, including ABR measurement, whereas adults, mostly with vestibular complaints, were evaluated in the laboratory of Dr. Coats. Imagine for a moment how the time course of pediatric audiology would have been altered if children coming to the audiology clinic in the early 1970s for diagnostic hearing assessment were evaluated with ECochG along with traditional hearing tests, plus the newly discovered ABR. It is very likely that the original crosscheck article would have included one or two case reports describing what we now refer to as auditory neuropathy spectrum disorder (ANSD). Instead, papers describing the collection of test findings associated with ANSD did not appear until almost 20 years later.

\section{Crosscheck principle defined simply}

Responding the implication in a popular pediatric audiology textbook that consistently accurate hearing assessment of children with behavioral techniques is rather straightforward, Jerger and Hayes acknowledge with characteristicly colorful and clear prose: "We are not so sanguine. We have found that simply observing the auditory behavior of children does not always yield an accurate description of hearing loss. In our own experience, we have seen too many children at all levels of functioning who have been misdiagnosed and mismanaged on the basis of behavioral results alone" [1]. Following the review of the 5 illustrative cases, the authors confidently conclude: "In summary, we believe that the unique limitations of conventional behavioral audiometry dictate the need for a "test battery" approach. The key concept governing our assessment strategy is the cross-check principle. The basic operation of this principle is that no result be accepted until it is confirmed by an independent measure" [1].

The pediatric test battery, and especially the inclusion of additional objective procedures, remained essentially unchanged until approximately 20 years later when otoacoustic emission (OAE) technology became widely available as a routine clinical procedure as multiple manufacturers marketed OAE devices and billing codes were approved in the USA. The Joint Committee on Infant Hearing (JCIH) in 2000 recommended strongly the routine application of ABRs elicited with frequency-specific tone burst stimulation, and also bone conduction ABR measurement as indicated, for auditory assessment of infants and young or difficult-to-test children. During the same time period, the ASSR emerged as a clinically feasible technique for objective estimation of auditory thresh- 
olds, especially in children with severe to profound hearing impairment. More recently ECochG has resurfaced once again as a valuable clinical tool, this time in the diagnosis of children with suspected ANSD. We now have readily available for use in patients of all ages, an assortment of objective techniques for early and accurate identification and diagnosis of every type and site auditory dysfunction, from middle ear disorders to ANSD to central auditory processing disorders (APDs), listed in Table 2.

Thousands of journal articles, hundreds of book chapters, and even entire textbooks, describe in detail the functional anatomy of objective auditory tests and how the procedures are performed and findings are analyzed. The remainder of this paper reviews briefly selected clinical advantages of four major objective auditory tests in the detection and diagnosis of infant hearing loss. It also highlights the unique contribution of each of these objective auditory procedures to the pediatric test battery.

Table 2. A modern test battery for pediatric auditory assessment

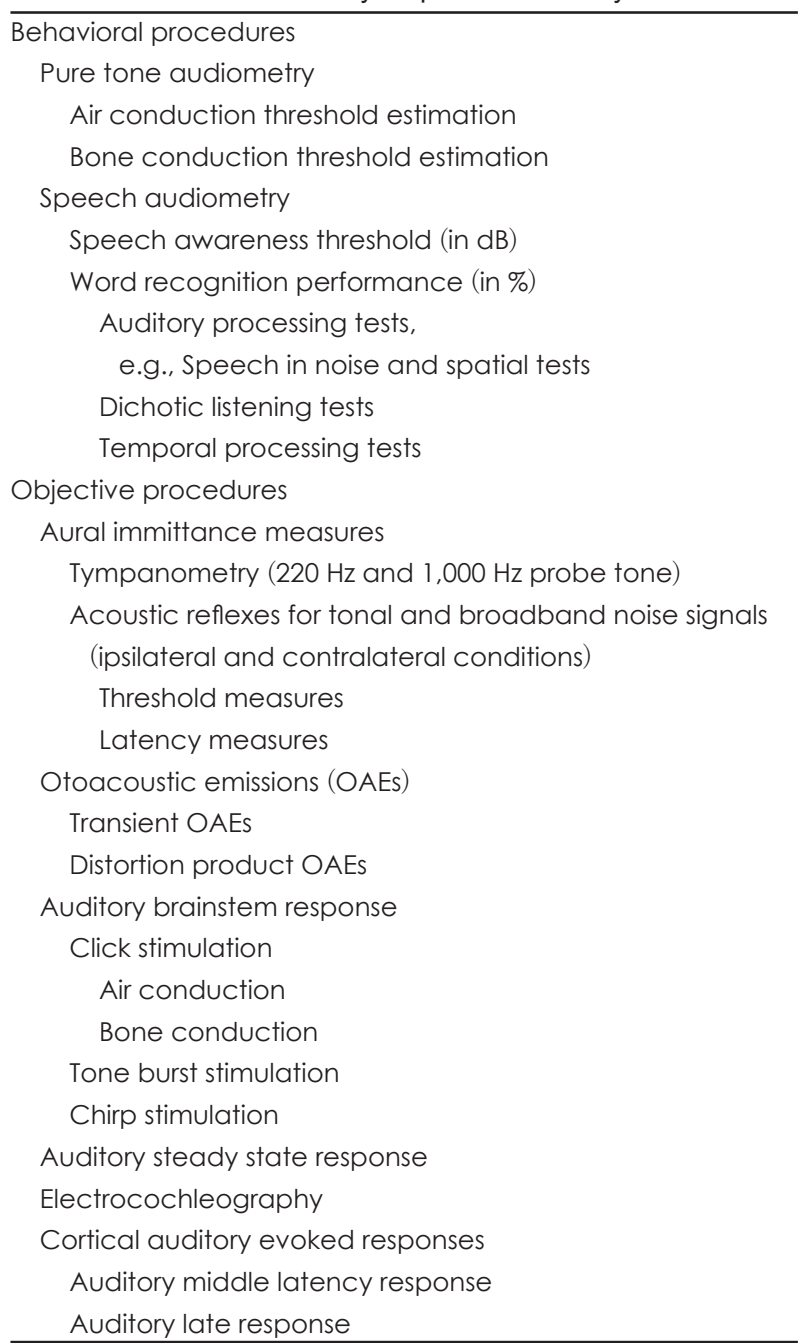

\section{Crosscheck Principle Today}

\section{Rationale for objective infant hearing assessment}

Policies, programs, and protocols for prompt identification, diagnosis, and management of hearing loss in children are now often referred to as Early Hearing Detection and Intervention (EHDI). The "1-3-6 Plan or Principle" is central to EHDI. That is, hearing loss is detected before 1 month, diagnosis of hearing loss is complete within the first 3 months after birth, and intervention begins within 6 months. Substantial research evidence supports the benefits of early intervention for the acquisition of effective and efficient communication skills along with psychosocial development [15]. It is now well known and appreciated that consistent and typical auditory stimulation within the first 6 months after birth and beyond is essential for proper central auditory nervous system development. The evolution of this rather accelerated schedule for EHDI greatly diminishes the role of behavioral hearing assessment in the initial diagnosis of hearing loss and the first habilitation efforts with hearing aids and other devices. Fortunately, the objective auditory tests listed in Table 2 are available for early and accurate diagnosis of infant hearing loss. Objective hearing procedures all share one major clinical advantage. They are not dependent on behavioral infant responses. Specific clinical strengths of objective hearing procedures are summarized in Table 3. Space does not permit a complete discussion of the many and varied clinical advantages of objective tests. Many articles and several books offer a thorough review of the rationale for the application of objective auditory tests in the assessment of infants and young children $[16,17]$. What follows here is a brief discussion of features of selected objective tests.

\section{Aural immittance measures}

\section{Introduction}

There are many clinical advantages specific to aural immittance measurements in pediatric patient populations. Indeed, "impedance audiometry" was a key component of the pediatric test battery described in the original crosscheck article. Then, with most clinical devices for aural immittance measurement it was possible to perform tympanometry with only a low frequency probe tone. Also, acoustic reflex measurement was limited to the contralateral test condition. Research since then has consistently confirmed the importance of including high frequency tympanometry in addition to lowfrequency tympanometry in infants up to the age of at least 4 months [16-18]. Aural immittance characteristics differ substantially for infants versus older children and adults. Specifi- 
Table 3. Clinical strengths of objective auditory measures available to audiologists (selected advantages associated with each test are highlighted within the text)

- Do not require a behavioral response from the patient

- Results are not influenced by motivation

- Results are not influenced by cognitive status

- Results generally are not influenced by state of arousal

- Measurements can be made with patient sedated or anesthetized

- Results are not influenced by native language

- Patient is not required to follow detailed verbal instructions

- Motor status does not influence test results

- Measures provide information on regions of the auditory system from the middle ear to the cerebral cortex

- Generally high degree of sensitivity to auditory dysfunction

- In combination provide site specific information on auditory dysfunction

- Valid measures are possible from infants and young children - Reasonable test time

cally, in comparison to older persons the middle ears of infants have a higher resistance component for a low frequency probe tone of $226 \mathrm{~Hz}$. Ear canal volume measurements in infants, however, should be conducted with a low frequency probe tone in children under the age of 6 months.

\section{The diagnostically powerful acoustic stapedial reflex}

The acoustic stapedial reflex is one of several muscle responses to sound. It falls in the same general category as the post-auricular muscle response, the eye blink reflex, and the startle response. Careful measurement of stapedial acoustic reflexes yields considerable information on the anatomical status of the auditory system, especially when recorded in four conditions, that is, measurement of ipsilateral and contralateral acoustic reflex activity with right and left ear stimulation.

Much of the auditory system is involved in the acoustic reflex measurement including the middle ear, the cochlea, the $8^{\text {th }}$ (auditory) cranial nerve on the side of the stimulus, brainstem neurons within the cochlear nuclei and, for contralateral acoustic reflex measurement, the trapezoid body and medial superior olivary complex plus polysynaptic pathways including neurons within the reticular activating system, an efferent pathway involving motor fibers within the $7^{\text {th }}$ cranial nerve on the side of the probe, plus the stapedius muscle and middle ear on the side of the probe. The presence of acoustic reflexes is highly dependent on normal middle ear function. Most middle ear abnormalities obscure confident detection of acoustic reflexes, even relatively subtle disorders that are not associated with markedly abnormal tympanograms or a significant $(>10 \mathrm{~dB})$ gap between air- and bone conduction pure tone thresholds.

Tympanometry has unquestionable value as a screening tool for the detection of middle ear abnormalities. However, hearing requires integrity of much more than the middle ear. The application of hearing loss estimation with acoustic reflex thresholds was first reported in the early 1970s [9]. Presuming normal middle ear function, acoustic reflexes permit quick, ear specific objective differentiation of normal versus abnormal cochlear function. The acoustic reflex threshold for broadband noise $(\mathrm{BBN})$ increases rather systematically with worsening pure tone thresholds for sensory hearing loss [20]. In contrast, acoustic reflexes elicited with pure tone signals showed little change in threshold from normal hearing sensitivity through 50 or even $60 \mathrm{~dB}$ HL, a reflection of the loudness recruitment phenomenon.

Fig. 1 illustrates the differential effect of sensory hearing loss on acoustic reflex thresholds elicited with tonal versus $\mathrm{BBN}$ signals. Patients with hearing loss rarely have acoustic reflex thresholds of less than $85 \mathrm{~dB}$ for the $\mathrm{BBN}$ stimulus. Conversely, BBN acoustic reflex thresholds greater than 90 $\mathrm{dB}$ are invariably associated with sensory hearing loss. Results from a study of acoustic reflex thresholds in neonates provides further support for the use of a BBN stimulus in objectively differentiating between normal hearing sensitivity versus sensory hearing loss. Kei [21] reported acoustic reflex threshold data collected with pure tone and BBN stimuli and a $1,000 \mathrm{~Hz}$ probe tone in a group of 66 health newborn infants who had passed hearing screening. Acoustic reflexes were recorded in all stimulus conditions from all of the infants. The median acoustic reflex threshold in the normal hearing infant group was $55 \mathrm{~dB}$ HL for the $\mathrm{BBN}$ stimulus with a range of 50 to $75 \mathrm{~dB}$ HL. These findings confirm that an acoustic reflex threshold of $75 \mathrm{~dB}$ HL or better is consistent with normal hearing sensitivity.

The value of acoustic reflexes in quickly and objectively differentiating among sites of auditory dysfunction is also impressive. There are four possible distinct and different measurement conditions in acoustic reflex measurement: 1) right ear ipsilateral, 2) left ear ipsilateral, 3) contralateral reflexes with the probe in the right ear and sound in the left ear, and 4) contralateral reflexes with the probe in the left ear and sound in the right ear. These four measurement conditions and normal findings for each condition can be displayed graphically in a diagram like the one shown in Fig. 2. An open box in the figure indicates the presence of normal acoustic reflexes with thresholds of $<90 \mathrm{~dB}$ HL. A shaded box indicates abnormally elevated acoustic reflex thresholds, whereas a filled in black box indicates that no acoustic reflex activity was detected in the test condition. 


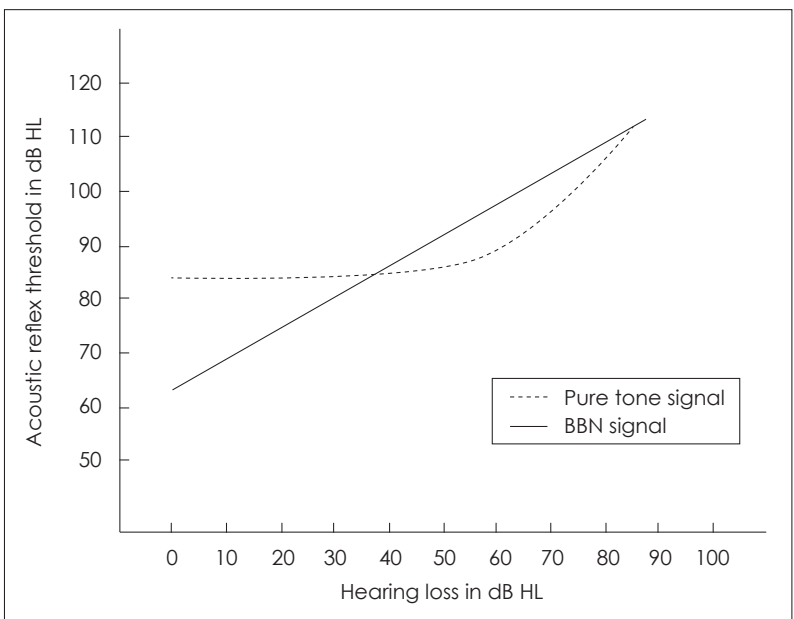

Fig. 1. Acoustic reflex thresholds for pure tone stimuli (solid line) versus BBN stimuli (dashed line) are depicted as a function of hearing threshold levels. BBN: broadband noise.

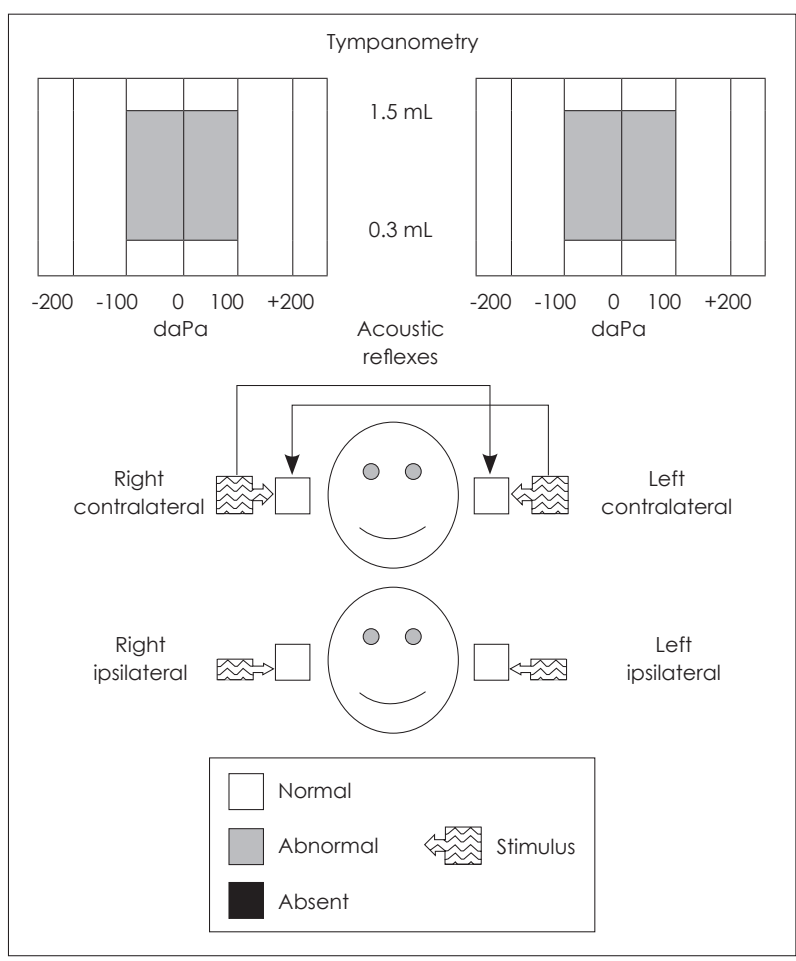

Fig. 2. A diagram for plotting findings for tympanometry and acoustic reflexes in the ipsilateral and contralateral conditions.

Combinations or patterns of findings for pure tone audiometry, tympanometry, and acoustic reflex recordings are generally related to likely clinical etiologies or diagnoses. In analysis of acoustic reflex patterns, it's useful to first examine the findings for tympanometry to confirm or rule out middle ear disorder followed by inspection of the acoustic reflex pattern for the four measurement conditions. An audiogram if available may offer additional evidence of conductive hearing loss. Several common acoustic reflex patterns are cited here.
A vertical pattern is often encountered clinically, particularly in pediatric populations where middle ear disorders are commonplace. Typically, acoustic reflexes are absent whenever the probe is on the side with middle ear dysfunction. Greater conductive hearing loss for the ear would result in elevation of the contralateral acoustic reflex measured with stimulation of that ear and the probe in the opposite normal ear. A conductive hearing loss essentially reduces the effectiveness of the acoustic reflex stimulation by the magnitude of air bone gap. Since the acoustic reflex is normally activated with an intensity level of $85 \mathrm{~dB} \mathrm{HL}$, a conductive loss of 25 to $30 \mathrm{~dB}$ raises the contralateral acoustic reflex threshold for stimulation of the ear with conductive hearing loss to about 110 to $115 \mathrm{~dB}$ HL. Facial nerve disorder is another explanation for the vertical pattern of acoustic reflex abnormality. Two factors clearly distinguish this vertical pattern from the acoustic reflex pattern typical of mild conductive hearing loss. The most obvious factor is normal tympanometry in facial nerve disorder, consistent with normal middle ear function. A normal audiogram or at least no difference between air and bone conduction pure tone thresholds also argues against middle ear disorder.

When acoustic reflexes are abnormally elevated in threshold or absent with stimulation of one ear, the most likely explanation is a sensory hearing loss. The chances of detecting acoustic reflex activity decline as the degree of sensory hearing loss increases. Normal acoustic reflex findings are anticipated in mild and even moderate sensory hearing loss, reflecting the loudness recruitment phenomenon. The acoustic reflex pattern in patients with unilateral neural dysfunction is similar, but the degree of hearing distinguishes it from sensory hearing loss. With neural auditory dysfunction secondary to an acoustic tumor such as a vestibular schwannoma, the diagonal acoustic reflex abnormality is often associated with only mild hearing loss. The neural pattern may also be suspected due to acoustic reflex decay.

Finally, a horizontal abnormal acoustic reflex pattern is sometimes encountered in patients with brainstem auditory dysfunction. The presence of normal ipsilateral acoustic reflexes and normal tympanometry unequivocally rule out conductive hearing loss, sensory hearing loss, neural auditory dysfunction, and facial nerve disorder. The horizontal acoustic reflex pattern is a strong sign of brainstem auditory dysfunction in patients at risk for central auditory nervous system dysfunction, including those with head injury and suspected APD.

Jerger, et al. [22] first described rare version of the horizontal pattern for acoustic reflex findings. The pattern is characterized by an abnormality is only one contralateral 
acoustic reflex condition. All pathologic explanations other than an isolated unilateral brainstem auditory abnormality are convincingly ruled out due to the presence of normal acoustic reflexes in the other three acoustic reflex conditions, plus normal tympanograms and typically normal hearing sensitivity bilaterally. Observation of the uni-box acoustic reflex abnormality prompts a comprehensive assessment of central auditory function and, in many cases, otolaryngology and/or neurology referral.

\section{Otoacoustic emissions}

\section{Multiple evidence-based applications of OAEs}

OAEs contribute importantly and in a truly unique way to the diagnosis of auditory dysfunction, even though they have essentially no value in defining the degree of hearing loss. OAEs are now an important component of the pediatric test battery and essential for implementation of the crosscheck principle. Standard of care for hearing assessment of infants and young children calls for routine measurement of OAEs [15]. In terms of anatomic site sensitivity and specificity, in particular the detection and verification of outer hair cell dysfunction, OAEs have no rival in the hearing test battery. Since their discovery in 1978, the literature on OAEs has continuously expanded [23]. There are now well over 4,000 peer reviewed articles on the topic. Selected evidence-based clinical applications of OAEs in children are listed in Table 4. In view of the sizeable and readily accessible information on OAEs, their role in the pediatric test battery will not be further reviewed here.

\section{Auditory evoked responses}

\section{Introduction}

Published literature on the multiple and varied applications of auditory evoked responses in clinical audiology is vast [6]. There is also remarkable accumulated clinical experience with electrophysiological responses elicited from the cochlea, auditory brainstem and auditory cerebral cortex. For example, we have witnessed a resurgence of interest in ECochG

Table 4. Selected evidence-based applications of otoacoustic emissions (OAEs) for pediatric patient populations

Newborn hearing screening

Diagnosis of auditory dysfunction in infants and young children

Differentiation of site of auditory dysfunction

Identification of auditory neuropathy spectrum disorder

Identification and diagnosis of false hearing loss

Monitoring ototoxicity

Pre-school and school-age screenings as a critical test in the accurate diagnosis of ANSD, in particular the differentiation of pre-synaptic inner hair cell versus post-synaptic neural sites of auditory dysfunction [24].

Auditory evoked responses are also useful in objectively evaluating function at the other end of the auditory system. Many thousands of papers confirm the value of tonal and speech evoked cortical evoked responses in objective assessment of central auditory nervous system function in diverse patient populations ranging from a variety neuropsychiatric diseases to children and adults with suspected APDs. One of the most recent and exciting applications of the auditory late responses is objective evaluation of cortical functioning in infants with sensory hearing impairment and ANSD undergoing habilitation with hearing aids and/or cochlear implants. The following brief review focuses exclusively on the ABR and the ASSR as components of the pediatric test battery and the current contribution of ABR and ASSR to the crosscheck principle.

\section{Auditory brainstem response}

Since Hecox and Galambos published their classic paper 1974, the ABR has remained unrivaled as a powerful diagnostic tool for in objective assessment of hearing in infants and young children [25]. A sizeable proportion of recent publications are devoted to innovative stimuli for eliciting ABRs, including chirps and complex stimuli like speech. The review that follows highlights the recent use of chirp stimuli in clinical measurement of the ABR in infants and young children. It is adapted from text found in the eHandbook of Auditory Evoked Responses [6].

There is consensus that the ABR evoked with conventional click stimulation is dominated by activation of the basal region of the cochlea. Attempts to enhance the contribution of other regions of the cochlea to ABR generation include the creation of rather unique types of stimuli called "chirps." Chirps are sounds that sweep rapidly from low-to-high frequencies or vice versa. The term chirp is derived from the sound that birds and some other animals produce. The chirp stimulus is designed mathematically to produce simultaneous activation along the cochlear partition by means of a compensation for traveling-time differences among frequency regions [26]. Chirp versions of click stimuli optimize synchronization across a broad frequency region at high and low intensity levels, yielding a more robust ABR than the conventional click stimulus.

The overall physiological goal with chirp stimuli is to simultaneously activate a wide range of the cochlea from base to apex, with resulting enhancement of ABR amplitude. Larger amplitude for ABR wave $\mathrm{V}$ is not a trivial goal. The significant clinical advantages of a larger wave $\mathrm{V}$, often a doubling 
in amplitude, and an increase in the ABR versus noise difference include: 1) more confident identification of wave $\mathrm{V}$ near the minimum response level or threshold, 2) detection of an $\mathrm{ABR}$ at lower intensity levels for presumably more accurate estimations of thresholds, and/or 3) decreased test time required for recording ABRs.

An additional comment about chirps is worth noting here. Chirp stimuli and their effectiveness in enhancing the amplitude of ABRs are dependent on specific mathematical formulae and models. Not all chirps are created the same way. The forgoing discussion focused mostly on CE-chirps developed by and described in the publications of Claus Elberling (CE) and colleagues who designed stimuli designated as "CEchirps" [27]. It's reasonable and advisable to inquire about the development of and clinical research evidence in support of chirps before applying them in ABR measurement from patients.

\section{Auditory steady state response}

Consistent with the cross check principle and in common with behavioral hearing tests and other electrophysiological auditory procedures, it is not advisable to record ASSR in isolation but, rather, as a component in an appropriate test battery. The literature reveals papers describing diagnostic applications of ASSR in various pediatric populations in addition to estimation of auditory thresholds. Importantly, ASSR and $\mathrm{ABR}$ are not competitive electrophysiological procedures. The decision clinically is not to record either tone burst ABR or ASSR. Rather, the two procedures are complementary. Diagnosis of hearing loss and plans for intervention are often based on results of some combination of ABR recordings and ASSR recordings in the same child, along with findings for other objective auditory tests.

The ASSR like the ABR offers an opportunity for estimation of auditory thresholds in infants and young children who cannot be properly assessed with behavioral audiometry techniques. The ASSR provides a distinct edge over behavioral audiometry and in several respects even the ABR in this clinically challenging patient population. One strong feature of the ASSR, in comparison to the ABR, is the capacity for defining severe to profound hearing loss, that is, estimating hearing thresholds within range of 80 to $120 \mathrm{~dB}$ HL. The limitation of $\mathrm{ABR}$ in defining the degree of severe-to-profound hearing loss $>90 \mathrm{~dB}$ HL is well appreciated by clinicians and well documented in the literature.

ABR and ASSR each can contribute importantly and rather uniquely to diagnostic auditory assessment of children. However, it is important, however, to keep in mind that neither the ABR nor the ASSR are actually tests of hearing. Each technique must be applied within an appropriate evidence based test battery consistent with the crosscheck principle [1] and with clinical guidelines for pediatric hearing assessment [15].

ASSR technology and techniques also continue to evolve. Papers are beginning to emerge describing comparison of chirp-evoked ASSR with hearing thresholds and with ABR thresholds [28]. Preliminary evidence suggests that chirpevoked ASSRs are equivalent to and perhaps superior to conventional ASSR techniques for quick and accurate estimation of behavioral thresholds in adults and, most importantly, in infants and young children with normal hearing or hearing impairment [29]. Efficiency and accuracy of technique chirpevoked ASSR appears to be related to increased amplitude. Very brief test times open up the possibility of performing ASSR assessments in reasonably cooperative infants and young children who are sleeping naturally without the assistance of sedation or anesthesia.

\section{Concluding Comments}

Forty years after it was first introduced, the crosscheck principle remains critically important in prompt and accurate diagnosis of hearing loss in children. The diagnostic power of objective auditory tests is only achieved when they are carefully selected and applied in a test battery. Exclusive reliance on only one or two tests, even objective auditory measures, often results in equivocal outcome and a diagnosis is not clear or perhaps incorrect. With an appropriately complete test battery, however, auditory disorders are invariably differentiated and described accurately.

Close analysis of findings for an objective auditory test battery almost always yields prompt and precise description of auditory status, and it often leads to accurate diagnosis of auditory dysfunction. Failure to apply the crosscheck principle in pediatric hearing assessment today will result in misdiagnosis and mismanagement of children resulting in unfortunate outcomes like those described by Jerger and Hayes in the 1976 article [1]. In summary, the application of a complete objective test battery is the most effective and efficient strategy for prompt and accurate diagnosis of auditory dysfunction and hearing loss in infants and young children. Objective auditory assessment is essential for a successful EHDI program. Hearing assessment with a collection of objective auditory tests defines standard of care in pediatric audiology.

\section{Conflicts of interest}

The author has no financial conflicts of interest. 


\section{REFERENCES}

1) Jerger JF, Hayes D. The cross-check principle in pediatric audiometry. Arch Otolaryngol 1976;102:614-20.

2) Jewett DL, Williston JS. Auditory-evoked far fields averaged from the scalp of humans. Brain 1971;94:681-96.

3) Jerger J, Mier M, Boshes B, Canter G. Auditory behavior in parkinsonism. Acta Otolaryngol 1960;52:541-50.

4) Jerger J, Golden D, Jerger S. Averaged evoked response audiometry by telemetry. 1968 IEEE National Telephone Conference Record, Winner, New York;1968. p.403-6.

5) Jerger J, Jerger $S$. Evoked response to intensity and frequency change. Arch Otolaryngol 1970;91:433-6.

6) Hall JW III. eHandbook of Auditory Evoked Responses: Principles, Procedures \& Protocols. Kindle Direct Publishing;2015. Available from: https://www.amazon.com/eHandbook-Auditory-Evoked-Responses-Principles-ebook/dp/B0145G2FFM.

7) Coats AC. Directional preponderance and unilateral weakness as observed in the electronystagmographic examination. Ann Otol Rhinol Laryngol 1965;74:655-68.

8) Coats AC. Directional preponderance and spontaneous nystagmus as observed in the electronystagmographic examination. Ann Otol Rhinol Laryngol 1966;75:1135-59.

9) Coats AC, Smith SY. Body position and the intensity of caloric nystagmus. Acta Otolaryngol 1967;63:515-32.

10) Coats AC. Central electronystagmographic abnormalities. Arch Otolaryngol 1970;92:43-53.

11) Coats AC. Human auditory nerve action potentials. Neurology 1970; 20:418.

12) Coats AC, Dickey JR. Nonsurgical recording of human auditory nerve action potentials and cochlear microphonics. Ann Otol Rhinol Laryngol 1970;79:844-52.

13) Coats AC. On electrocochleographic electrode design. J Acoust Soc Am 1974;56:708-11.

14) Coats AC. The summating potential and Meniere's disease. I. Summating potential amplitude in Meniere and non-Meniere ears. Arch Otolaryngol 1981;107:199-208.

15) American Academy of Pediatrics, Joint Committee on Infant Hearing. Year 2007 position statement: Principles and guidelines for early hearing detection and intervention programs. Pediatrics 2007;120:
898-921.

16) Hall JW, Swanepoel D. Objective assessment of hearing. San Diego: Plural Publishing;2010

17) Hall JW. Introduction to audiology today. Boston: Pearson;2014.

18) Hall JW. Aural immittance measures in audiology: more useful now than ever before. Hear J 2010;63:10-5.

19) Jerger J, Burney P, Mauldin L, Crump B. Predicting hearing loss from the acoustic reflex. J Speech Hear Disord 1974;39:11-22.

20) Hall JW 3rd, Berry GA, Olson K. Identification of serious hearing loss with acoustic reflex data. Clinical experience with some new guidelines. Scand Audiol 1982;11:251-5.

21) Kei J. Acoustic stapedial reflexes in healthy neonates: normative data and test-retest reliability. J Am Acad Audiol 2012;23:46-56.

22) Jerger $S$, Jerger J, Hall J. A new acoustic reflex pattern. Arch Otolaryngol 1979;105:24-8.

23) Dhar S, Hall JW. Otoacoustic Emissons: Principles, Procedures, and Protocols. San Diego, CA: Plural Publishing;2011.

24) McMahon CM, Patuzzi RB, Gibson WP, Sanli H. Frequency-specific electrocochleography indicates that presynaptic and postsynaptic mechanisms of auditory neuropathy exist. Ear Hear 2008;29: 314-25.

25) Hecox K, Galambos R. Brain stem auditory evoked responses in human infants and adults. Arch Otolaryngol 1974;99:30-3.

26) Fobel O, Dau T. Searching for the optimal stimulus eliciting auditory brainstem responses in humans. J Acoust Soc Am 2004;116(4 Pt 1):2213-22.

27) Elberling C, Don M. Auditory brainstem responses to a chirp stimulus designed from derived-band latencies in normal-hearing subjects. J Acoust Soc Am 2008;124:3022-37.

28) Venail F, Artaud JP, Blanchet C, Uziel A, Mondain M. Refining the audiological assessment in children using narrow-band CE-Chirpevoked auditory steady state responses. Int J Audiol 2015;54:106-13.

29) Mühler R, Mentzel K, Verhey J. Fast hearing-threshold estimation using multiple auditory steady-state responses with narrow-band chirps and adaptive stimulus patterns. ScientificWorldJournal 2012;2012: 192178.

30) Swanepoel de W, Hall JW III. A systematic review of telehealth applications in audiology. Telemed J E Health 2010;16:181-200. 